\title{
Die Corona-Pandemie und das Grundgesetz - Anmerkungen nicht nur zu Rechtsfragen
}

\section{FRANZ KNIEPS}

Franz Knieps ist Volljurist und Vorstand des BKK Dachverbandes e.V.; Knieps ist Mitherausgeber der Zeitschrift „Gesundheitsund Sozialpolitik“*

Die Bekämpfung der Pandemie durch SARS-CoV-2/Covid-19 stellt Staat, Wirtschaft, Gesellschaft und Institutionen aber auch alle Bürgerinnen und Bürger vor bisher unbekannte Herausforderungen. Für das Gesundheitswesen wirft sie eine Vielzahl von Fragen auf, die von grundsätzlicher politischer Bedeutung und für die alltägliche Versorgungspraxis relevant sind. Derzeit ist es sicherlich zu früh, konkrete Schlussfolgerungen für die künftige Ausgestaltung des Gesundheitswesens zu ziehen. Der folgende Beitrag setzt den Schwerpunkt - entsprechend der Primärqualifikation des Verfassers - bei den Rechtsfragen. Er stützt sich dabei ganz wesentlich auf die gemeinsame Arbeit von Wissenschaftlern und Praktikern, die die politische und operative Arbeit bei der Bekämpfung der Pandemie kritisch begleiten (Schrappe et al. 2020 a und b), auch wenn die politisch Verantwortlichen in Bund und Ländern keinesfalls von Kritik begeistert waren.

\section{Zeiten der Unsicherheit - Die Situation bei Ausbruch der Epidemie}

SARS-Cov-2/Covid-19 ist eine Infektionskrankheit mit hoher Infektiosität und sehr verschiedenen Krankheitsverläufen. Besonders gefährdet sind ältere Menschen und Menschen mit Vorerkrankungen. Die meisten Infizierten zeigen gar keine Symptome oder solche, die einem grippalen Infekt gleichen. Dies trägt dazu bei, dass Infektionsherde erst spät oder gar nicht erkannt werden. Auch Einrichtungen des Gesundheitsund Sozialwesens können solche Herde bilden. Zudem besteht die Gefahr, dass diese - wie Berichte aus Italien, Frankreich, Großbritannien oder den USA zeigen - sehr schnell an die Grenzen ihrer Belastbarkeit kommen können. Trotz eines generell gut ausgestatteten Gesundheitssystems und einer gewissen Vorlaufzeit von den ersten Meldungen aus der Volksrepublik China bis zum Ausbruch der Epidemie in Deutschland, offenbarten sich schnell Ausstattungsund Koordinationsprobleme. Speziell in der ambulanten ärztlichen und zahnärztlichen Versorgung und noch gravierender in der pflegerischen Versorgung machte sich ein Mangel von adäquater Schutzausrüstung und ein Fehlen von Mitarbeitenden bemerkbar. Zumindest kurzfristig kam das ambulante

* Der Beitrag gibt allein die persönliche Auffassung des Autors wieder. 
Versorgungssystem zum Erliegen, die stationäre Versorgung wurde kurzfristig umgestaltet, um nicht - wie ebenfalls in anderen Ländern zu beobachten - vor dem Ansturm von Menschen mit schweren Krankheitsverläufen kapitulieren zu müssen. Zumindest in der ersten Welle ist die befürchtete Überlastung des Versorgungssystems glücklicherweise nicht eingetreten. Im Gegenteil, der überwiegende Teil der kurzfristig eingerichteten Intensivbetten ist nicht belegt. Die übrige Krankenhausversorgung und speziell die ambulante Versorgung verzeichnen starke Rückgänge, die mit der „normalen“ Krankheitslast nicht erklärbar sind und zum Teil auf unterbliebene Behandlungen mit ungewissen gesundheitlichen und sozialen Folgen schließen lassen.

\section{Präventionsmaßnahmen zur Weiterentwicklung der Bekämpfungsstrategien}

Angesichts der unvertretbaren Folgen bei der Entwicklung eines Herden-Immunität und der Weiterverfolgung der Strategie der Abflachung der Ausbreitungskurve stellt sich die Frage, ob bis zur Entwicklung eines Impfstoffes und der Durchimpfung der Bevölkerung die allgemein-präventiven Maßnahmen mit drastischen Kollateralschäden nicht durch spezifische Präventionskonzepte zum Schutz der Risikogruppen ergänzt und schrittweise abgelöst werden müssen. Die Autorengruppe um Matthias Schrappe (Schrappe et al. 2020 b) hat hierzu ein spezifisches Konzept entwickelt, das bei den Risiken hohes Alter, Komorbidität, nosokomiale Risiken und Kontakt mit Infektionsherden (Clustern) ansetzt und nicht auf Abgrenzung zielt, sondern verbesserten Schutz vor einer Virusinfektion zum Ziel hat. Zur Bestimmung des Risikos schlägt die Gruppe ein mehrdimensionales Scoring-Modell vor. Zudem spricht sie sich nachdrücklich für eine spezifische Berücksichtigung der Belange von Kindern und Jugendlichen, von Menschen mit Behinderungen oder in spezifischen sozialen Lebenslagen (z.B. Obdachlose) sowie der im Gesundheitsund Sozialwesen einschließlich der ehrenamtlich oder im Familienverbund tätigen Menschen aus. Schließlich empfiehlt sie den flächendeckenden Aufbau sog. Corona-Task Forces zur temporären Unterstützung der Verantwortlichen vor Ort. Diese Task Forces sollen und können allerdings nicht verdecken, dass die Bundesländer den öffentlichen Gesundheitsdienst sträflich vernachlässigt und wie bei der Investitionsfinanzierung stationärer Einrichtungen kläglich versagt haben.

\section{Verfassung und Recht unter den Bedingungen von Unsicherheit}

Nach einer anfangs zögerlichen Haltung haben sich Bundes- und Landesregierungen auch unter dem Eindruck der Entwicklung in Asien und anderen europäischen Ländern zu beispiellosen Maßnahmen entschlossen, die das öffentliche Leben in Deutschland weitgehend zum Erliegen brachten und unabsehbare wirtschaftliche und soziale Folgen nach sich ziehen. Diese drastischen Maßnahmen werfen eine Vielzahl von juristischen Fragen auf. Diese betreffen das Staatsorganisationsrecht der Bundesrepublik ebenso wie die Grundrechte unserer Verfassung. Besondere Aufmerksamkeit findet dabei das am 28.3.2020 in Kraft getretene, im Eilverfahren verabschiedete und inzwischen erweiterte Gesetz zum Schutz der Bevölkerung bei einer epidemischen Lage von nationaler Tragweite (BGBl. 2020 I 587), das zu einer erheblichen Veränderung des bisher geltenden Infektionsschutzgesetzes (IfSG) geführt hat (Einzelheiten bei Rixen 2020; Klafki 2020). Durch die Veränderungen werden einerseits die Kompetenzen des Bundes ausgeweitet, obwohl die Bundesländer das Gesetz - wie andere Bundesgesetze auch - in eigener Verantwortung ausführen. Andererseits werden die Länder ermächtigt, eigene Schutzmaßnahmen in Form von Rechtsverordnungen zu erlassen $(\mathbb{S} 32$ IfSG).

\section{Die Stunde der Exekutive - Im Grundgesetz unbekannt}

Dabei ist es von eminenter politischer und verfassungsrechtlicher Bedeutung, dass die Neufassung des Infektionsschutzgesetzes die Gewichte zwischen Legislative und Exekutive verschiebt. Diese Verschiebung stößt auf erhebliche Bedenken (exemplarisch Bäcker 2020; Klafki,2020). Zwar hat der Deutsche Bundestag im Gesetzgebungsverfahren durchgesetzt, dass das Parlament selbst das Vorliegen einer epidemischen Lage von nationaler Tragweite feststellt und diese Feststellung auch wieder aufhebt. Doch nach dieser Feststellung werden dem Bundesminister für Gesundheit weitreichende Ermächtigungen zum Erlass von Rechtsverordnungen - in der Regel ohne Beteiligung des Bundesrats - und zu sofort vollziehbaren Anordnungen erteilt. Diese Ermächtigungen gestatten dem BMG Modifikationen und Ausnahmen von nahezu allen gesetzlichen und untergesetzlichen Bestimmungen des öffentlich-rechtlichen Gesundheitsrechts einschließlich der Rechtsetzung durch Selbstverwaltung ( 55 Abs. 2 InfSG). Nach der Regelung wird das BMG ermächtigt, sogar von jeder Befugnis des IfSG und darauf gestützter Verordnungen abzuweichen, um die Abläufe im Gesundheitswesen und die Versorgung der Bevölkerung aufrecht zu erhalten. Spätestens hier halten selbst Grenzen des Artikel 80 Abs. 1 Satz 2 GG hinsichtlich der Bestimmung von Inhalt, Zweck und Ausmaß für überschritten (Rixen NJW 2020, 1097, 1102 f.). Aber auch für alle anderen Ermächtigungsnormen gilt, dass sie mit äußerster $\mathrm{Zu}$ rückhaltung auszulegen und anzuwenden sind, vor allem wenn und soweit sie in Grundrechte eingreifen.

Das Grundgesetz kennt keine Stunde der Exekutive (Möllers 2020 b; Kaiser 2020; Schönberger 2020). Auch vermeiden es Gerichte, Politiker und die Mehrheit der Juristen, von einem Ausnahmezustand zu sprechen (So aber unter Bezugnahme auf die Diskussion um den sog. Präventionsstaat Volkmann 2020, differenzierend Mehring 2020). Auch will der überwiegende Teil nichts vom alten Grundsatz „Not kennt kein Gebot" wissen (anders aber wohl Isensee 2020). Politisch fatal wäre es, sich auf den umstrittenen Juristen und Rechtsphilosophen Carl Schmitt (1888-1985) und sein Diktum in dessen Politischer Theologie zu berufen: „Souverän ist, wer über den Ausnahmezustand entscheidet" (Schmitt 1922, 9). Vielmehr mehren sich nach einigen Wochen die Stimmen, die die unter Demokratie- und 
Gewaltenteilungsaspekten folgenschwere Selbstentmachtung des Parlaments zugunsten einer nahezu schrankenlosen Dominanz der Exekutive sehr kritisch sehen (exemplarisch Kingreen 2020). Gerade in Krisenzeiten haben die Bürgerinnen und Bürger, die demokratischen Entscheidungen Respekt und Loyalität zollen, einen Anspruch darauf, dass der Staat in allen Fragen von Legitimation und Legalität penibel alle Formen wahrt sowie Kritik demokratisch aufnimmt und nicht mit Illoyalität verwechselt. „Der Notfall, den es zu bekämpfen gilt, bedarf der Überprüfung; die Maßnahmen, die er rechtfertigen soll, umso mehr“ (Möllers 2020 a). „Beispiellose Freiheitseingriffe bedürfen beispielloser Transparenz" (Spamann 2020). Es bedarf also in schwierigen politischen Zeiten kontroverser Debatten im öffentlichen Raum, um sich gerade im Kontext von Unsicherheiten (Frankenberg 2020) nicht der Illusion der Alternativlosigkeit von Zielen und Maßnahmen hinzugeben.

Weitgehende juristische Einigkeit besteht auch hinsichtlich der Bewertung von in der Krise zuerst ergriffenen Maßnahmen, mögen sich diese auch in der Form und in der konkreten Ausgestaltung von Land zu Land oder sogar mit regionalen oder örtlichen Besonderheiten unterscheiden. Parlamenten und Regierungen wurde zugutegehalten, dass sie trotz der Erfahrungen mit früheren Epidemien und der zumindest theoretisch durchgespielten Pandemieplanung von der Wucht der Erkrankung überrascht wurden.

Die Gerichte billigen der Exekutive einen breiten Ermächtigungs- und Beurteilungsspielraum bei der Einschätzung der Gefahrenlage sowie bei der Auswahl und Ausgestaltung von Maßnahmen zur Eindämmung der Seuche und zur Begrenzung der Ansteckungsrisiken zu (exemplarisch VGH München, NJW 2020, 1236, 1240: OVG Bautzen, NJW 2020, 1384 ff.). Dies gilt vor allem für Maßnahmen zu Beginn der Pandemie. Je länger Regelungen, insbesondere solche mit Grundrechtseinschränkungen, schon in Kraft sind, umso stärker wirkt die Verpflichtung zur kontinuierlichen Überprüfung der Geeignetheit und der Verhältnismäßigkeit. An diese Prüfung sind strenge Maßstäbe anzulegen (BVerfG, NJW 2020, 1429). Selbst wenn eine Maßnahme von vornherein zeitlich befristet ist, besteht eine Pflicht zur regelmäßigen Evaluierung, insbesondere im Hinblick auf die Erforderlichkeit und die Angemessenheit. Sollte sich die Unverhältnismäßigkeit einzelner Regelungen herausstellen, sind diese isoliert unverzüglich auch vor Ablauf des Geltungszeitraums eines Gesamtpakets aufzuheben.

\section{Grundrechte gegen Grundrechte - Die hohe Kunst der Abwägung}

Schließlich besteht auch Einigkeit, dass viele der von Bund und Ländern erlassenen Rechtsakte erhebliche Eingriffe in wesentliche Freiheitsrechte der Menschen in Deutschland bedeuten. In diese Grundrechte darf durch Gesetz oder auf Grund eines Gesetzes eingegriffen werden. Generell werden nur wenig Zweifel geäußert, dass die Regelungen des Infektionsschutzgesetzes, insbesondere die $\int \mathbb{S} 28$ Abs. 1 und 32 des IfSG als ausreichende Rechtsgrundlagen für Verordnungen, Allgemeinverfügungen und Einzelverfügungen angesehen werden (BVerfG, NJW 2020: 1429; Wissenschaftlicher Dienst 2020, 15).

Ganz wesentlich kommt es für die Beurteilung der Rechtmäßigkeit auf die Verhältnismäßigkeit der Eingriffe an. Diese Prüfung darf nicht mit der „Holzhammermethode“ (Hofmann 2020) er-

\section{Für die Beurteilung von Grundrechtseingriffen kommt es auf die Verhältnismäßigkeit}

an.

folgen. Jeder Eingriff muss ein legitimes Ziel in geeigneter, erforderlicher und angemessener Weise verfolgen. Kohärenz ist notwendige „Begleitmusik zum infektionsschutzrechtlichen Tanz" (Michl 2020). Unzweifelhaft sind der Schutz der Bevölkerung und die Verhinderung der Überlastung des Gesundheitswesens legitime Ziele für Grundrechtseingriffe. Auch dürften Maßnahmen zur Verringerung oder gar Verhinderung von sozialen Kontakten grundsätzlich geeignet sein, die Infektionsgeschwindigkeit des Virus zu verlangsamen oder die Pandemie zum Stillstand zu bringen. Bei der Auswahl der Maßnahmen besteht — zumindest zu Beginn einer Pandemie — ein weiter
Beurteilungsspielraum. Dabei gilt der allgemeine Grundsatz der Gefahrenabwehr, dass an die Wahrscheinlichkeit des Schadenseintritts umso geringere Anforderungen zu stellen sind, je größer und folgenschwerer die Schäden sein können. Inwieweit Ausweispflichten, die Untersagung der Nutzung von Zweitwohnungen oder das Verbot von Reisen geeignet sind, legitime Ziele des Infektionsschutzes zu erreichen, darf mehr als bezweifelt werden (Wissenschaftlicher Dienst 2020, 16 f. mit Nachweisen aus der Rechtsprechung). Auch landesweite Ausgehverbote dürften eine Verhälnismäßigkeitsprüfung kaum überstehen (Schmitt 2020. Das gilt erst recht für das Verbot, die Grenzen eines Bundeslands zu überschreiten, oder gar das Gebot, ein Bundesland zu verlassen. Dieses Verhalten eines Landes, das primär vom Tourismus lebt, markiert einen Tiefpunkt des föderalen Egoismus. Die $\mathrm{Ab}$ - und Ausweisung an Ländergrenzen sollte im Jahr 30 der Wiedervereinigung politisch und juristisch tabu sein. Das gilt besonders für den Fall, dass Bürger aus weniger betroffenen Landesteilen in Landesteile mit höherer Krankheitslast ausgewiesen werden, nur weil sie dort mit ihrem Erstwohnsitz gemeldet sind. Eine andere Landesregierung war cleverer und hat die Anreise zu einem Zweitwohnungssitz unabhängig von der Lage ihres Erstwohnsitzes untersagt. Das zuständige Oberverwaltungsgericht hat in einer Güterabwägung zugunsten des öffentlichen Interesses an einer Eindämmung der Pandemie entschieden (OVG Schleswig, NJW 2020, 1382ff.). Dagegen haben das OVG Berlin-Brandenburg (NJW 2020, 1454ff.) wie schon die Vorinstanz (VG Potsdam, COVuR 1/2020, 64f. und 65ff.) Zweifel an der Geeignetheit und der Verhältnismäßigkeit einer entsprechenden Reisebeschränkung durch Allgemeinverfügung eines Landkreises artikuliert und deshalb diese Verfügung aufgehoben. Berliner durften deshalb ihre Datschen in Brandenburg weiter nutzen.

Trefflich streiten lässt sich darüber, ob die vielen Einschränkungen in allen Einzelfällen erforderlich oder ob nicht andere weniger belastende Maßnahmen verfügbar sind (Lepsius 2020 beklagt in diesem Zusammenhang einen Niedergang grundrechtlicher Denkkategorien). 
In diesem Zusammenhang ist zu prüfen, wann spezifische Präventionsmaßnahmen für besonders gefährdete Risikogruppen allgemeine Gebote und Verbote abmildern oder ersetzen können (Huster 2020). Vor allem aber ist mit zunehmender Dauer der Freiheitsbeschränkungen immer wieder zu prüfen, ob diese nicht außer Verhältnis zum verfolgten Zweck stehen (Berliner Verfassungsgerichtshof, NJW 2020, 1505 ff.). Bei der Prüfung reicht es nicht aus, allein auf die Erkrankungs- und Todeszahlen abzustellen. Das Grundrecht auf Leben und körperliche Unversehrtheit begründet sicher besondere Schutzpflichten des Staates, ist aber nicht per se anderen Grundrechten übergeordnet. Vor allem ist es nicht zulässig, Leben gegen Leben aufzurechnen und abzuwägen. Die diesbezüglichen Ausführungen des Bundesverfassungsgerichts zur Nichtigkeit einer Abschussermächtigung im Luftsicherheitsgesetz (BVerfGE 115, 118 ff.) sind Pflichtlektüre für alle, die (verfassungs-)rechtliche Überlegungen zur Corona-Pandemie anstellen. Wie sonst wäre es zu erklären, dass auf Autobahnen kein Tempolimit gilt (Kingreen 2020).

Bei anhaltendem Verlauf der Pandemie wird kontinuierlich zu prüfen sein, inwieweit Ausnahmeregelungen von strikten Regulierungen ausgeweitet und die Zielsetzung der Regelungen durch Auflagen mit milderen Vorgaben (Abstandsgebot, Maskenpflicht ...) erreicht werden kann. Daraus lässt sich aber keine allgemeine Regel für alle denkbaren Fallgestaltungen ableiten, sondern „nur“ die allgemeine Verpflichtung, für jede Fallkonstellation eine Abwägung konkurrierender Grundrechte im Kontext des jeweiligen Erkenntniszustands vorzunehmen. Was gestern noch unausweichlich war, kann schon morgen fehlerhaft sein. Diese Anforderungen gelten im Übrigen nicht nur für bereits beschlossene Maßnahmen, sondern erst recht für das, was in Erwägung gezogen wird. Das betrifft etwa die diskutierte Ausstellung von lmmunitätsausweisen oder den Einsatz von Tracing- und Tracking-Apps, deren Zulässigkeit von der technischen Ausgestaltung (zentrale oder dezentrale Speicherung der Daten) und der Freiwilligkeit der Anwendung abhängig sein wird. Auch dürfte eine Rolle spielen, ob die Daten gegen den Gebrauch für fremde Zwecke, zum Beispiel für die Strafverfolgung, geschützt sind (Kühling/Schildbach 2020).

\section{Lockerungsübungen - Der schwierige Weg in die Differenzierung}

Die verfassungsrechtlichen Problemstellungen sind besonders relevant bei der Beurteilung von Maßnahmen zur Lockerung der Beschränkungen. Diese müssen insbesondere dem Prüfmaßstab des allgemeinen Gleichheitssatzes des Artikel 3 Absatz 1 GG entsprechen. Danach darf weder wesentlich Gleiches willkürlich ungleich noch wesentlich Ungleiches willkürlich gleichbehandelt werden (ständige Rechtsprechung des Bundesverfassungsgerichts seit BVerfGE 1, 14, 16); 4, 144, 155). Das Gericht differenziert dabei zwischen Ungleichbehandlungen geringerer und größerer Intensität. Die bisher verfügten Einschränkungen und deren Ausnahmen bieten eine Fülle von Vergleichsmöglichkeiten (Beispiele beim Wissenschaftlichen Dienst 2020, 31 ff.). Diese beschäftigen bereits die Rechtsprechung, gegebenenfalls wie bei der Öffnung von Ladenlokalen mit einer Verkaufsfläche bis zu 800 Quadratmetern mit unterschiedlichem Ausgang. So ist es folgerichtig, dass das Bundesverfassungsgericht das ausnahmslose Verbot von Gottesdiensten mit Gläubigen vor Ort gekippt hat (Pressemitteilung Nr. 28a/2020 vom 29. April 2020), nachdem es noch einige Wochen zuvor die Durchführung von Ostergottesdiensten abgelehnt hatte (BVerfG NJW 2020, 1427 ff,). Auch das ausnahmslose Demonstrationsverbot wurde im Laufe der Zeit durch das höchste Gericht gelockert (BVerfG, NJW 2020, 1426 f.; NJW 2020, 1505). Zudem sehen Landesverfassungsgerichte jetzt genauer hin als zu Beginn der Pandemie (Heumann/Holzgang 2020) und treten obrigkeitsstaatlichen Tendenzen in der Krise entgegen (Kumm 2020).

Liegt eine Ungleichbehandlung innerhalb einer Vergleichsgruppe vor, kann diese gleichwohl gerechtfertigt sein, wenn das Differenzierungsziel legitim ist, die Differenzierungskriterien zur Erreichung des Ziels geeignet sind und die Differenzierung erforderlich und angemessen ist. Diese Prüfung steht vor allem dann an, wenn die Politik spezielle Maßnahmen für besondere Risikogruppen verhängen sollte. Insbesondere das Kriterium Alter dürfte verfassungsrechtlich sehr unterschiedlich bewertet werden, selbst wenn das Grundgesetz, anders als diverse internationale Konventionen, das Alter nicht als besonderen Schutztatbestand vor Diskriminierung benennt. Ausdrücklich genannt ist in Artikel 3 Absatz 3 GG der Tatbestand der Behinderung, dem bisher nur begrenzte Aufmerksamkeit gewidmet wird. Schließlich werden auch Besuchsverbote und -einschränkungen in Alters- und Pflegeheimen zunehmend schwerer zu legitimieren sein (Glaab/ Schwedler 2020).

Hier kommt das oberste Prinzip der Verfassung ins Spiel, der Schutz der Menschenwürde in der Einleitungsvorschrift des Artikel 1 Absatz 1 Satz 1 GG. Aus diesem Leitprinzip der Verfassung leitet das Bundesverfassungsgericht die Voraussetzung ab, dass der Mensch in seiner fragilen und individuellen und sozialen Existenz über sich nach ei-

\section{Maßnahmen zur Lockerung von Beschränkungen sind verfassungsrechtlich relevant.}

genen Maßstäben verfügen kann und nicht in Lebensformen gedrängt werden darf, die in einem unauflösbaren Widerspruch zum eigenen Selbstbild und Selbstverständnis stehen (BVerfG, NJW 2020, 905 ff.). Der Mensch darf dabei nicht zu einem Objekt staatlichen Handels herabgewürdigt werden. Viele der ergriffenen Maßnahmen, speziell die noch weitgehend unerforschten gesundheitlichen und sozialen Wirkungen, bergen zumindest das Potenzial dazu (Wissenschaftler Dienst 2020, 37 f.). Auf der anderen Seite leitet das Gericht aus der Menschenwürde in Verbindung mit dem Grundrecht auf Leben und körperliche Unversehrtheit eine staatliche Schutzpflicht für das schwache Leben ab (BVerfGE 88, 203, 251 f.). Konkret sind also die freie und gleiche Persönlichkeitsentfaltung in Würde mit der Würde der besonders gefährdeten Risikogruppen abzuwägen (Kube 2020 und wohl auch Huster 2020). Daraus ergibt sich schon sachlogisch, dass es gerade in diesen Konstellationen keine rechtmäßigen „Pauschalmaßnahmen“ geben kann. Ein allgemeines Sonderopfer bestimmter Gruppen für die Volksgesundheit kommt nicht oder nur in sehr engen Grenzen in Betracht (Albers 2020). Erst recht kann 
ein Mensch mit Vorerkrankungen nicht verlangen, dass eine Anlaufpraxis zum Test auf das Coronavirus in der Nachbarschaft geschlossen wird (VG Bremen, COVuR 1/2020, 53ff.).

Mit zunehmender Dauer von Zuständigkeitsverlagerungen und Grundrechtseinschränkungen steigt der Druck, die Maßnahmen nicht nur auf eine zuverlässige epidemiologische Grundlage zu stellen, sondern auch zu transparenten und nachvollziehbaren Abwägungsprozessen zurückzukehren, die rechtsstaatlichen Maßstäben genügen. Das Bundesverfassungsgericht hat in fast 70 Jahren seiner Geschichte klare Entscheidungen getroffen und die Rechtswissenschaft hat vielfältige Orientierungshilfen gegeben (Heinig/Schorkopf 2019; Lepsius 2020). Not kennt also durchaus Gebot (Kaiser 2020, 207ff.).

\section{Reformen bleiben auf der politischen Tagesordnung}

Die Covid19-Pandemie wird Wirtschaft und Gesellschaft, den Staat und das Sozialleben grundlegend verändern (beispielhaft Ulrich 2020). Das bedeutet natürlich auch, dass Strukturen und Prozesse im Gesundheitswesen auf den Prüfstand kommen werden. Die zentralen Fragen nach Zielbild, Organisation, Steuerung und Finanzierung des Gesundheitswesens bleiben auf der Tagesordnung, müssen aber ergänzt werden um eine systematische Analyse der Stärken und Schwächen, der Chancen und Risiken des deutschen Gesundheitswesens. Wer - wie der Hauptgeschäftsführer der Deutschen Krankenhausgesellschaft - jubelt, nun sei eine Strukturbereinigung der deutschen Versorgungslandschaft obsolet, hat nicht nur nichts gelernt aus dieser Krise, sondern erweist sich als unfähig, die Zukunft zu gestalten.

\footnotetext{
** Angesichts der (noch) spärlichen Literaturlage greift der Verfasser viele Artikel und Anmerkungen aus dem Netz auf.
}

\section{Literatur}

Albers, G: Sonderopfer für die

Volksgesundheit: Freiheitsbeschränkungen zum Lebensschutz in der Coronakrise, VerfBlog vom 11.5.2020, https://verfassungsblog.de/ sonderopfer-fuer-die-volksgesundheit/

Bäcker, (2020): Corona in Karlsruhe II, Verfassungsblog vom 8.4.2020, https:// verfassungsblog.de/corona-in-karlsruhe/

Frankenberg, G.: COVID-19 und der juristische Umgang mit Unsicherheit, Verfassungsblog vom 25.4.2020, https://verfassungsblog.de/covid-19und-der-juristische-umgang-mit-unsicherheit/

\section{Glaab, S./Schwedler, A.:}

Besuchseinschränkungen wegen Corona in Pflegheimen, NJW 2020, 1702 ff.

Heinig, H. M./Schorkopf, F. (Hrsg.): 70 Jahre Grundgesetz - In welcher Verfassung ist die Bundesrepublik?, Göttingen 2019

Heumann, M./Holzgang,M.: Die Stunde der Verfassungsgerichte, Verfassungsblog vom 30.4.2020, https://verfassungsblog.de/diestunde-der-verfassungsgerichte/

Hofmann, C.M.: Verhältnismäßigkeit mit der Holzhammermethode, Verfassungsblog om 13.4.2020, https://verfassungsblog. de/verhaeltnismaessigkeit-mit-derholzhammermethode/

Huster, S.: Grenzen der Solidarität, Verfassungsblog vom 22.3.2020,https:// verfassungsblog.de/grenzen-der-solidaritaet/

Isennsee, J.: Virokratie im Rechtsstaat, FAZ vom 5.6.2020

Kaiser, A-B.: Ausnahmeverfassungsrecht, Tübingen 2020

Katzenmeier, C.: Grundrechte in Zeiten von Corona - Zugleich Anmerkung zu BVerfG, Beschl. vom 7.4.2020 - 1 BvR 755/20, MedR (2020) 38, https://doi.org./10.1007/s00350-020-5561-8

Klafki, A.: Neue Rechtsgrundlagen im Kampf gegen Covid-19, Verfassungsblog vom 25.3.2020, https://verfassungsblog.de/neuerechtsgrundlagen-im-kampf-gegen-covid-19/

Kingreen, T.: Whatever it takes? Der demokratische Rechtsstaat in Zeiten von Corona, Verfassungsblog vom 20.3.2020, http://verfassungsblog.de/whatever-it-takes/

Kube, H.: Leben in Würde - Würde des Lebens, Verfassungsblog vom 2. April 2020, http:// verfassungsblog/leben-in-wuerde-wuerdedes-lebens/

Kühling, J./Schildbach, R.: Corona-Apps - Datenund Grundrechtsschutz, NJW 2020, 1545 ff.

Kumm, M.: Gegen obrigkeitsstaatliche Tendenzen in der Krise, Verfassungsblog vom 20.4.2020, https://verfassungsblog.de/gegen obrigkeitsstaatliche-tendenzen-in der-krise/

Lepsius, O.: Vom Niedergang grundrechtlicher Denkkategorien in der Corona-Pandemie, Verfassungsblog vom 6.4.2020, https:// verfassungsblog.de/vom-niederganggrundrechtlicher-denkkategorien-in-dercorona-epidemie/

Mehring, R.: Carl Schmitt und die Pandemie, Teile I und II, VerfBlog vom 11.5 und 12.5.2020, https://verfassungsblo.de/carl-schmitt-und-diepandemie-teil-i bzw. teil-ii/

Michl, W..: Die Kohärenz als Begleitmusik zum infektionsschutzrechtlichen Tanz, Verfassungsblog vom 28.4. https:// verfassungsblog.de/die-kohaerenz-alsbegleitmusik-zum-infektionsschutzrechtlichen$\operatorname{tanz}$

Möllers, C. (2020 a): Über den Schutz der Parlamente vor sich selbst in der Krise, Verfassungsblog vom 20.3.2020, https:// verfassungsblog.de/über-den-schutz-derparlamente-vor-sich-selbst-in-der-krise/

Möllers, C. (2020 b): Parlamentarische Selbstentmächtigung im Zeichen des Virus, Verfassungsblog vom 27.3.20200 (b), https:verfassungsblog.de/parlamentarischeselbstentmächtigung.im-zeichen-des-virus/

Rixen, S.: Gesundheitsschutz in der Coronavirus-Krise - Die (Neu-) Regelungen des Infektionsschutzgesetzes, Neue Juristische Wochenschrift 2020, $1097 \mathrm{ff}$.

Schmitt, C.: Politische Theologie - Vier Kapitel zur Lehre von der Souveränität, Berlin 1922

Schmitt, F.: Verfassungswidrigkeit landesweiter Ausgehverbote, NJW 2020, 1626 ff.

Schönberger, S.: Die Stunde der Politik, Verfassungsblog vom 29.3.2020, httsp:// verfassungsblog.de/die-stunde-der-politik

Schrappe, M./Francois-Kettner, H./Gruhl, M./ Knieps, F./Pfaff, H./Glaeske, G. (Schrappe et al. 2020 a): Datenbasis verbessern - Prävention gezielt weiterentwickeln - Bürgerrechte bewahren, Thesenpapier 1.0, Monitor Versorgungsforschung 03/2020, $53 \mathrm{ff}$

Schrappe, M./Francois-Kettner, H./Knieps, F./ Pfaff, H./Püschel, K./Glaeske, G. (Schrappe et al. 2020 b): Datenbasis verbessern - Prävention gezielt weiterentwickeln - Bürgerrechte bewahren, Thesenpapier 2.0, Monitor Versorgungsforschung 03/2020, $64 \mathrm{ff}$.

Spamann, H.: Beispiellose Freiheitseingriffe brauchen beispielloseTransparenz, Verfassungsblog vom 4.4.2020, http://verfassungsblog.de/ beispiellose-freiheitseingriffe-brauchenbeispiellose-transparenz/

Thielbörger, P./Behlert, B. (2020 a): COVID-19 und das Grundgesetz, Verfassungsblog vom 19.3.2020, https://verfassungsblog.de/covid-19und-das-grundgesetz/

Thielbörger, P/Behlert, B. (2020 b): Covid-19 und das Grundgesetz: Neue Gedanken vor dem Hintergrund neuerGesetze, Verfassungsblog vom 30.3.2020, https://verfassungsblog.de/ covid-19-und-das-grundgesetz-neue-gedankenvor-dem-hintergrund-neuer-gesetze/

Ulrich, B.: Die desinfizierte Gesellschaft, Die Zeit Nr.22 vom 20.5.2020

Volkmann, U.: Der Ausnahmezustand, Verfassungsblog vom 20.3.2020, https:// verfassungsblog.de/der-ausnahmezustand/

Wissenschaftlicher Dienst des Deutschen Bundestags, Kontaktbeschränkungen zwecks Infektionsschutz: Grundrechte, WD 3-3000 079/20, Berlin 2020 